\title{
استخدامات الخط العربي وأثره النفعي والجمالي
}

\section{قسم التربية الفنية - كلية التربية الأساسية دولة الكويت}

إعداد/ أ. مشبب عبيد شباب العتيبيا

مقدمة :

شرفت اللغة العربية من بين اللغات العالمية بأنها اللغة التي أنزل الله بها القرآن الكريم على محمد صلى الله عليه وسلم

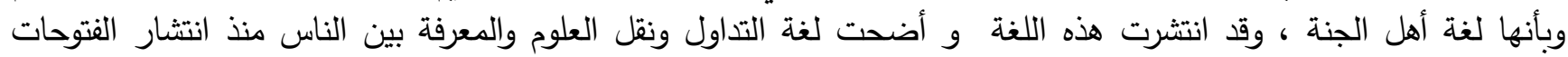

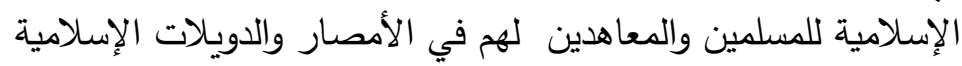

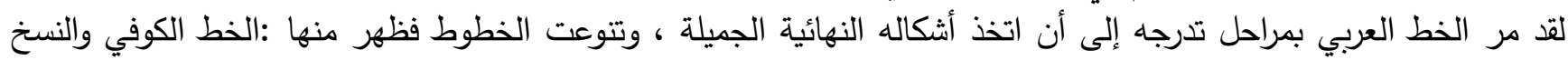

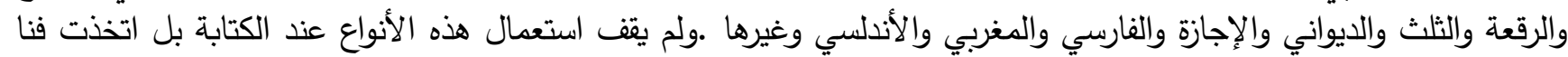

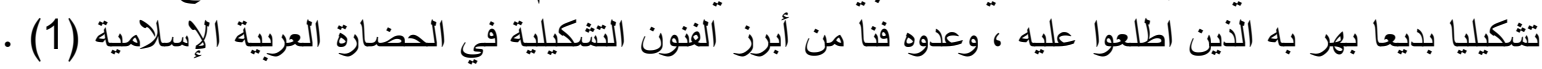

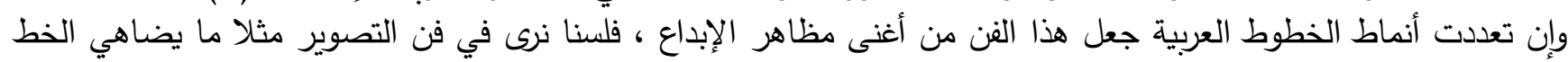

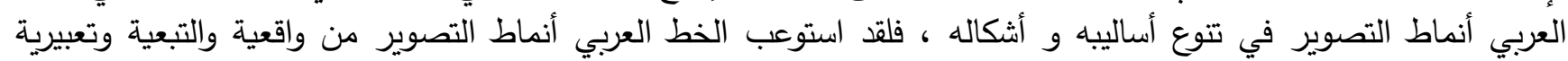

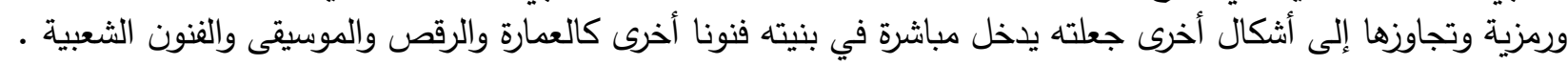

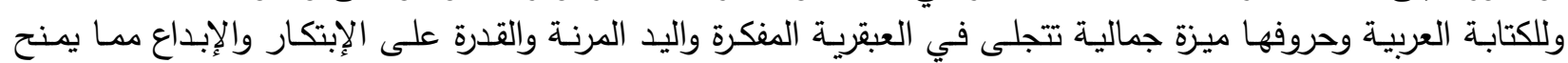

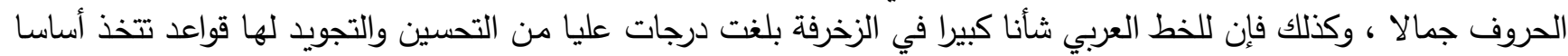

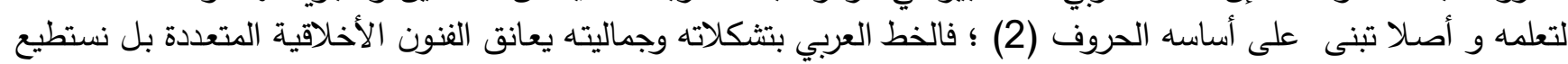

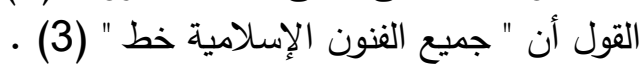
ويقوم الباحث بعمل دراسة تركز على التعريف بأنواع الخطوط العربية وأكثرها استخداما في الأعمال الفنية والجمالية وتبيان طرق المنفعة في استخدامها في مجتمعاتنا و في حياتتا اليومية.

\section{الدراسات السابقة :}

تتاولت دراسـة فاديه محمد هشام (4) ابراز قيم الجمال بين الأصـالة والمعاصرة من خلال استخدام الخط العربي من قبل

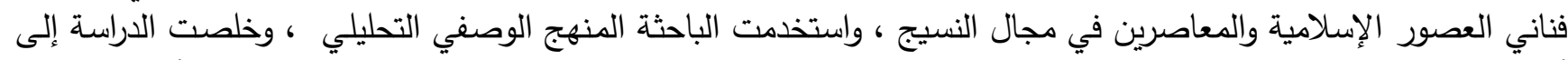

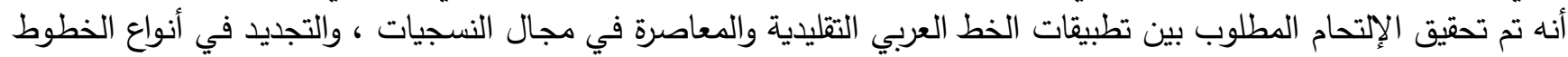

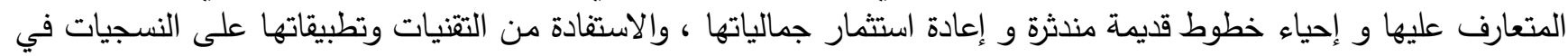

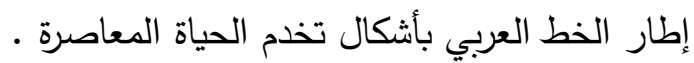

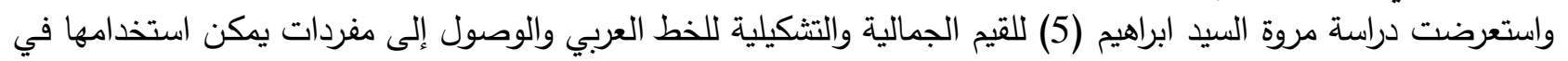

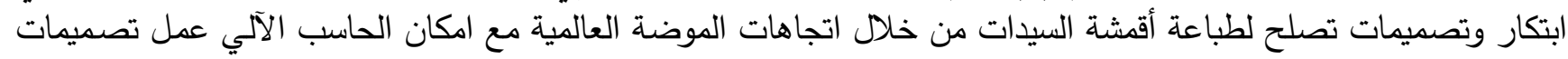

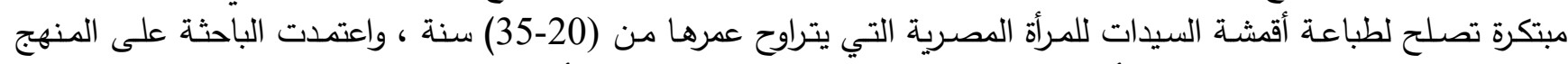

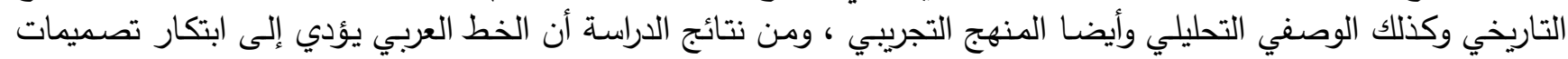

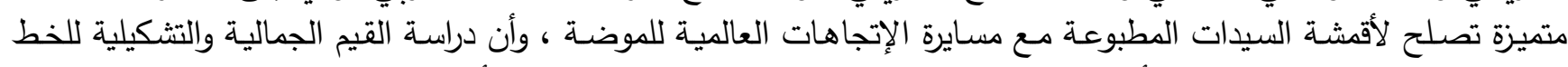

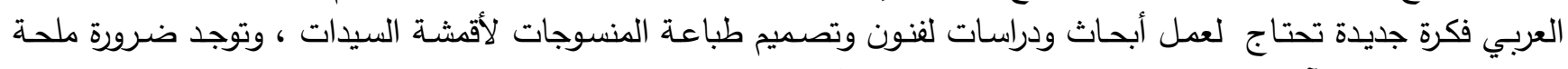

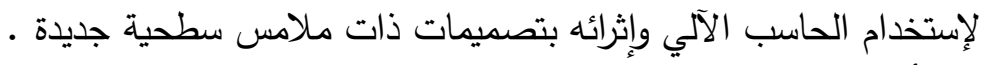

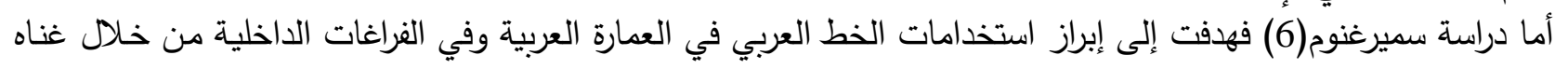

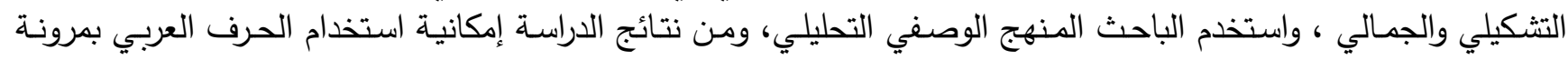

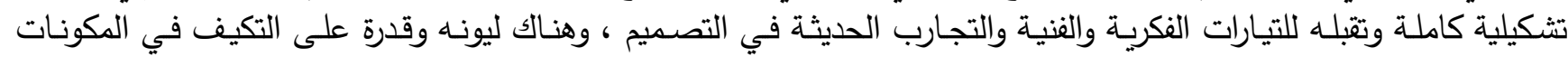

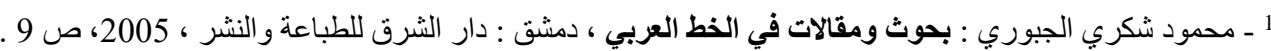

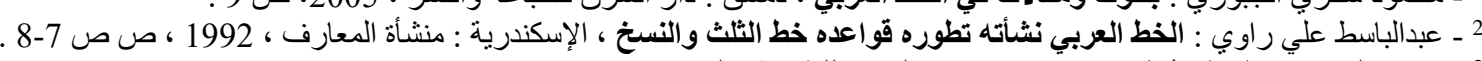

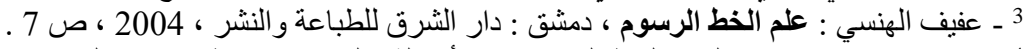

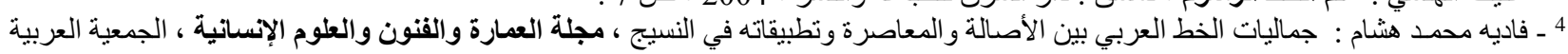

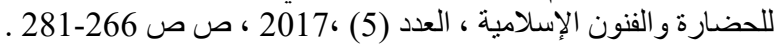

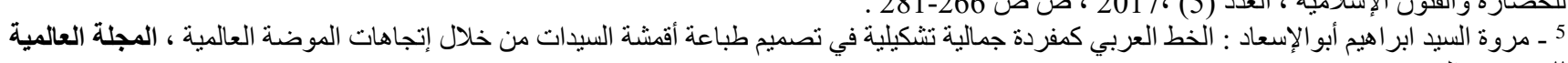

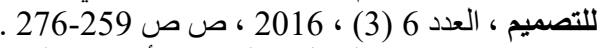
6 - سمير غنوم ، العدد 6 (3) ، الية الخط العربي ، وتأثيره في العمارة و إغناء الفراغ الداخلي ، مجلة جامعة دمشث للعلوم الهندسية ، العدد الثاني ، 2014 ، ص ص 147- 
الهندسية للحرف العربي ما يجعله يحقق هدفين الأول ظاهري في إيصال المفاهيم والتعابير والثاني تشكيلي في تكيفه مع أشكال

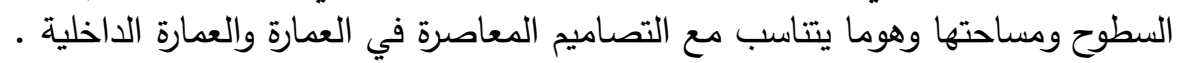

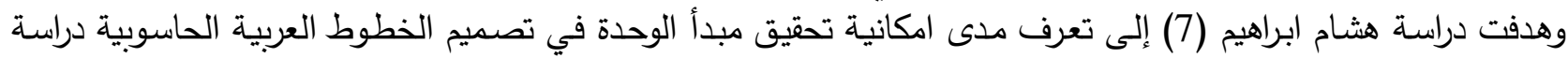

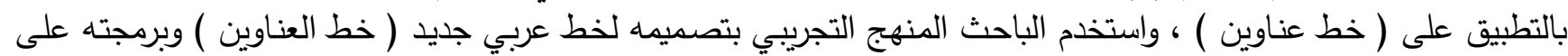

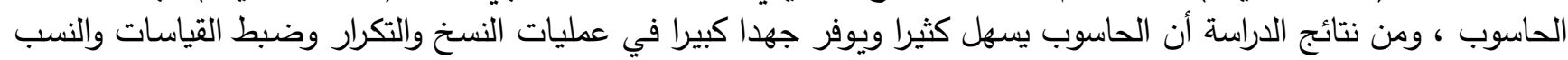

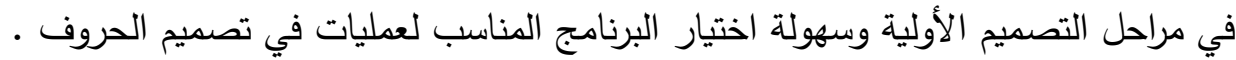

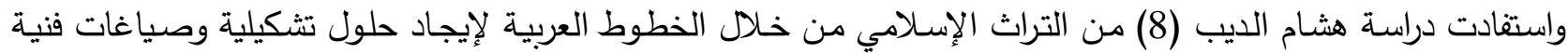

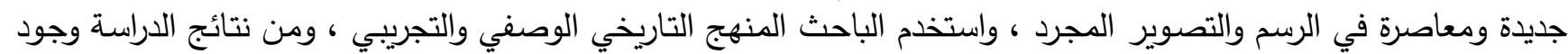

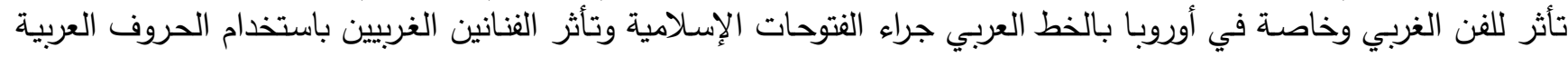

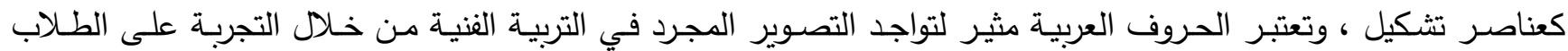

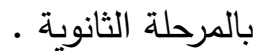

مشكالة الدراسة :

تكمن مشكلة الدراسة في كون الباحث لديه مساهمات فنية كثيرة في تصميم لوحات فنية تحتوي على تشكيلات من الخوط

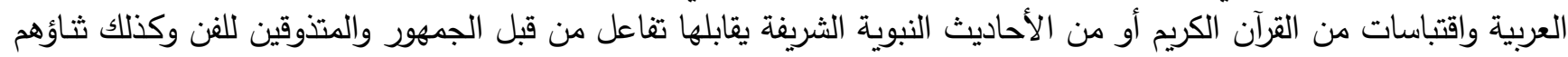

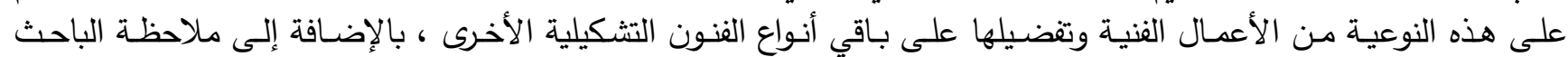

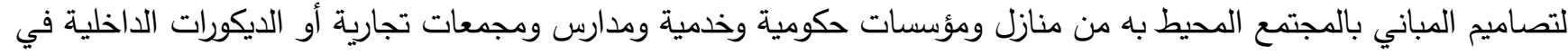

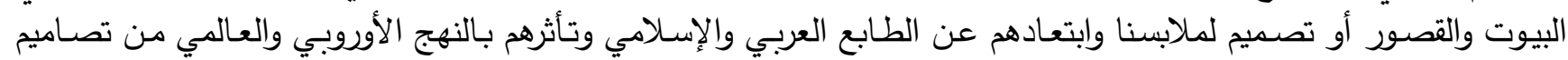

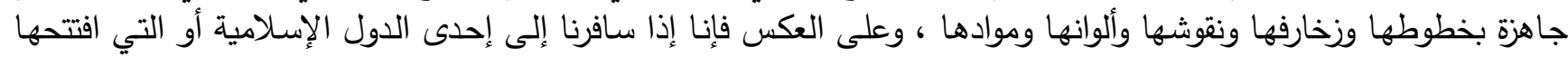

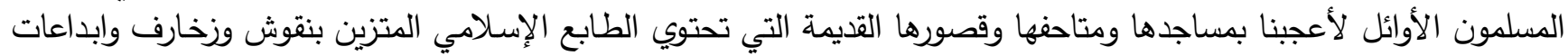

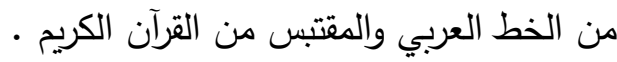
أسئلة الاراستة : يمكن تحديد مشكلة الدراسة في الأنس الأسئلة الاتية :

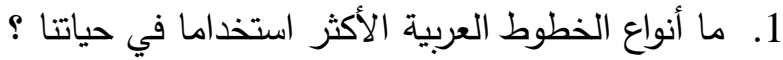

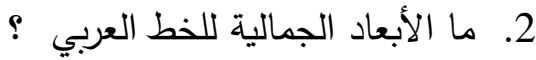

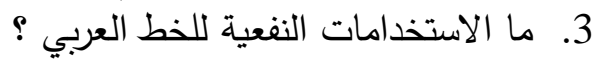

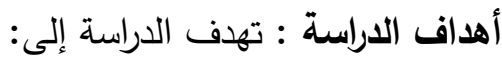

1 - تحديد أكثر أنواع الخطوط العربية العافية استخداما في حياتتا .

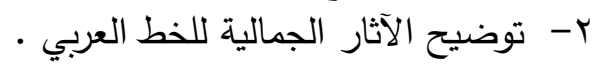
ץ- تعرف الاستخدامات التي يمكن الانتفاع منها باستخدام الخط العربي .

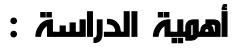

تكمن أهمية الدراسـة في إبراز أثر الخط العربي الجمالي والمنفعي من خـلال التعريف بأنواع مختلفة من الخطوط العربية

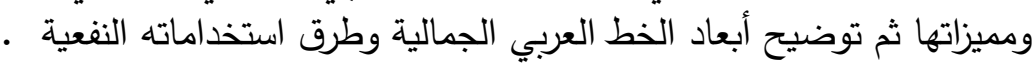

منهح الدراسة :

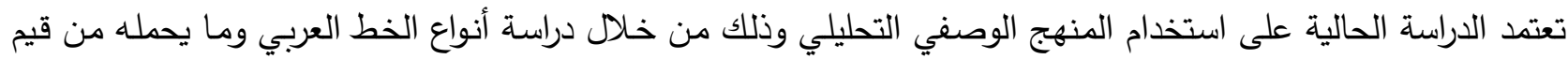

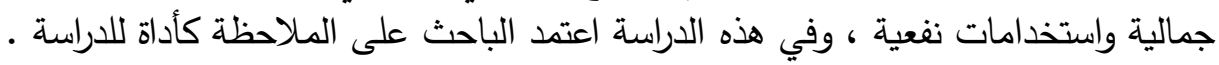

حدود الدراسة :

- تقتصر الدراسة على تحديد استخدامات الخط العربي النفعية والجمالية . - تقتصر الدراسة على ملاحظة طلاب مقرر ( فنون جميلة ) بقسم التربية الفنية في كلية التربية الأساسية بدولة الكويت ،

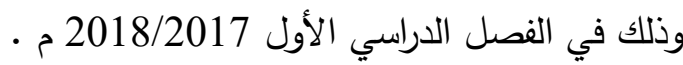

اجراءات الدراسة :

تسعى هذه الدراسة في جانبها النظري إلى التعريف بأكثر أنواع الخطوط العربية استخداما في حياتتا اليومية ، ثم في الخطوة الثانية يتم تحديد مجموعة من الأبعاد الجمالية للخط العربي ، ثم يتم في الخطوة الثالثة الكثف عن الاستخدامات النفعية للخط

7 ـ ـ هثام ابر اهيم عز الدين : امكانية تحقيق مبدأ الوحدة في تصميم الخطوط العربية الحاسوبية دراسة بالتطبيق على ( خط عناوين ) ، مجلة العلوم الإنسانية ،

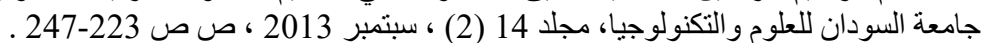

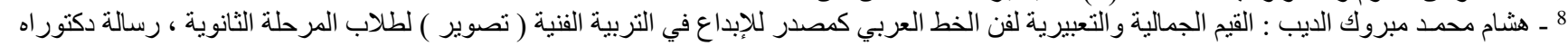
، كلية التربية النو عية قسم التربية الفنبة تخصص تصوير ، ، جامعة القاهرة ، 2005 ـ 
العربي ـ أمسا في جانبها الميداني فيتم عرض خمسة نماذج على طلبة مقرر ( فنون جميلة ) ويتم ملاحظة مستوى أدائهم في

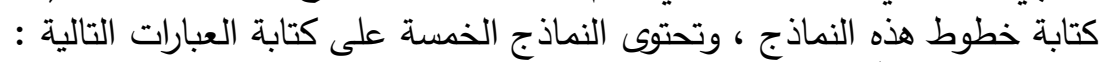

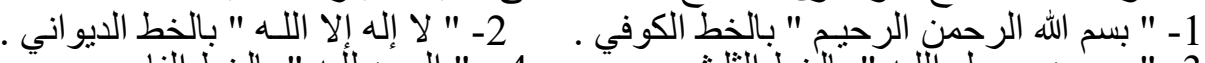

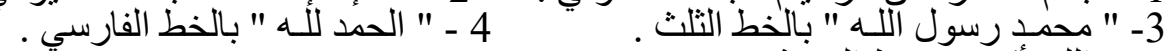

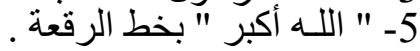

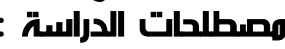

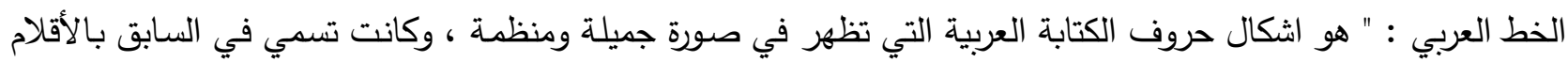

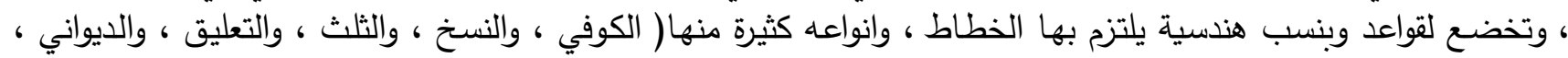

(9) الرقعة ) (

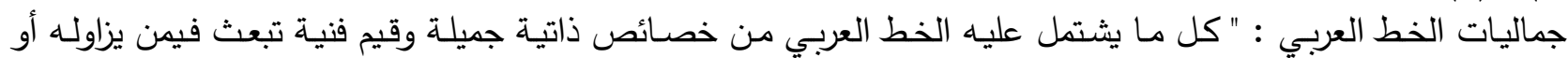

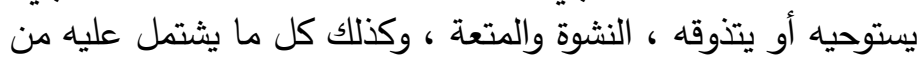

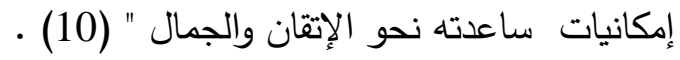

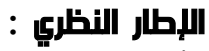

أولا : أنواع الخط العربي : عرف العاني الخط بأنه (11) " فن لرسم الحروف الهجائية، والتعبير عن الثكل والمضمون بأصول، وقواعد هندسية زخرفيه

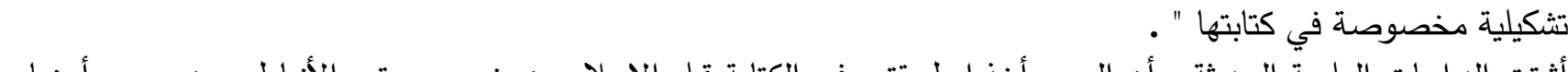
أثبتت الدراسات العلمية الحديثة ، أن العرب أخذوا طريقتهم في الكتابة قبل الإسلام من بني عمومتهم الأنباط ، وهم عرب أيضا ،

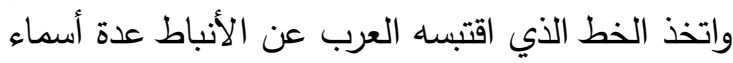

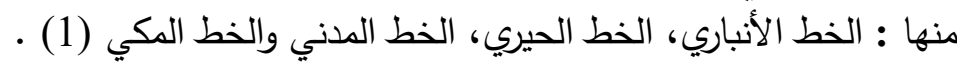

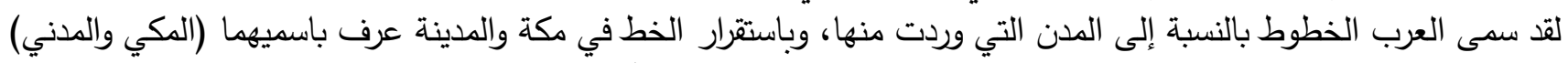

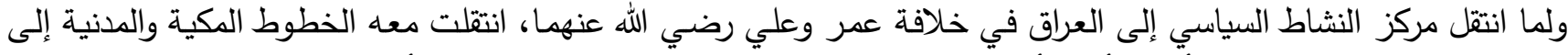

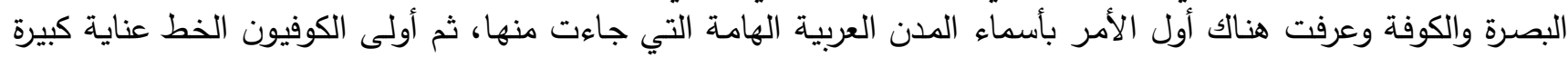

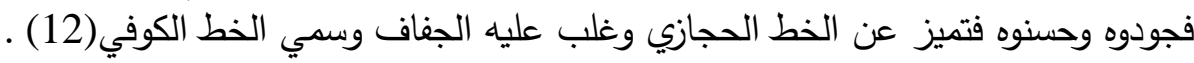
وفي القرن الثالث الهجري لما كثرت أعداد الخطوط وتتوعت أشكُالها ظهرت الحاجة إلى تركيز أنواعها وتصفية المتشابه منها

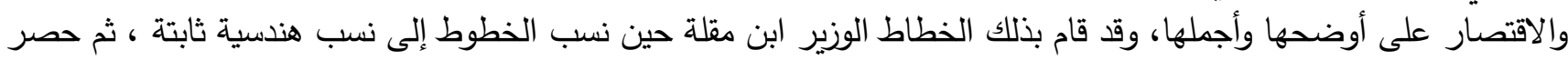

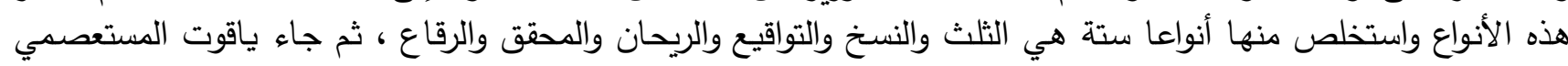

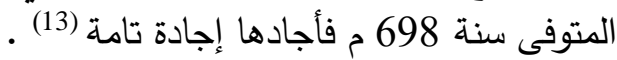
والخطوط العربية الأكثر انتشارا حاليا هيا أ- الخط الكوفي :

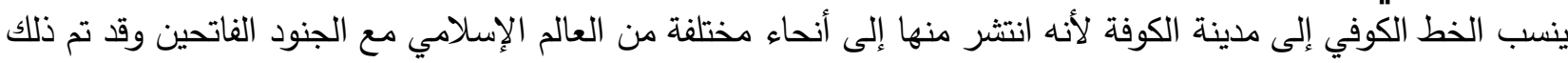

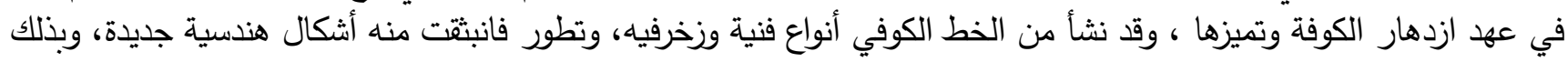

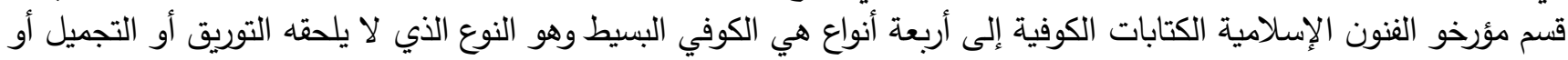

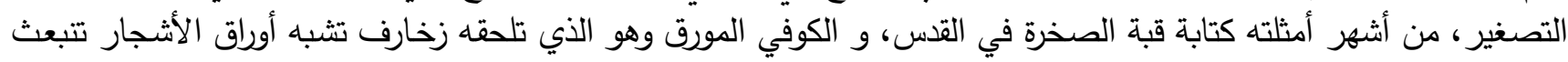

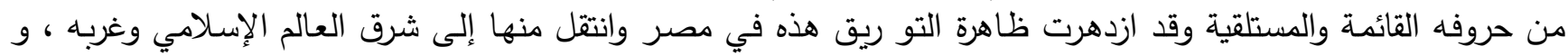

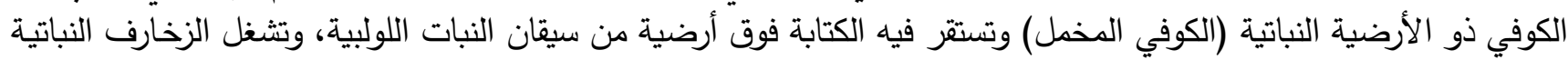

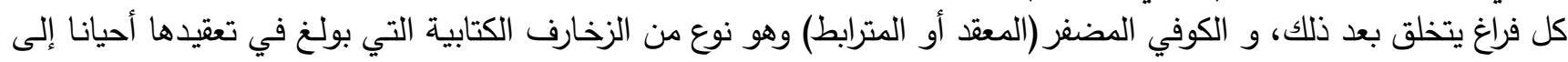

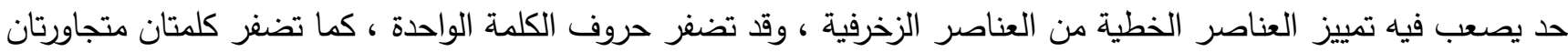

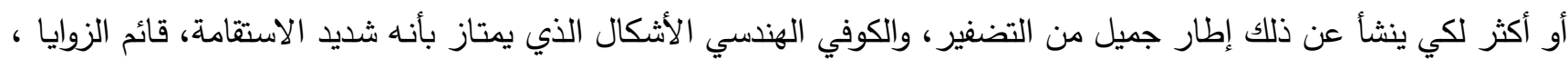

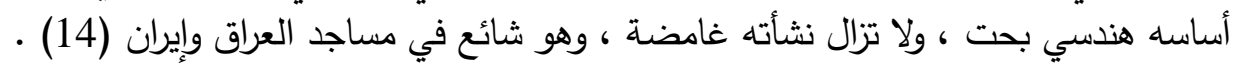

9 ـ عبد المحسن حسين عبد الرضا شيشتر: الوظيفة الزخرفية للحرف العربي كمدل تجريبي لتدريس التصميم في التربية الفنية ، رسالة ماجستير ، كلية

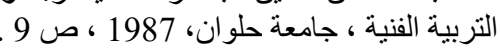

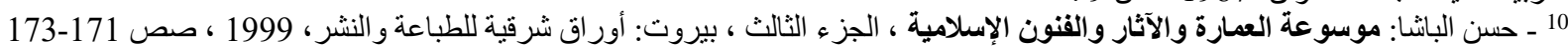

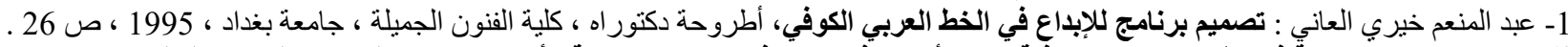

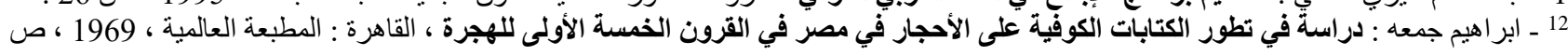

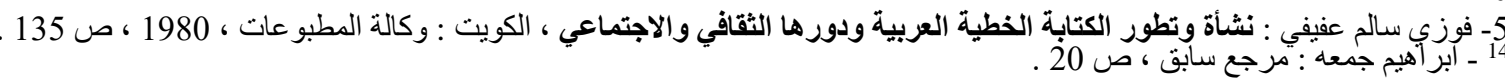




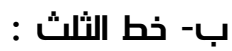

يعبر عنه بأنه سيد الخطوط ، فلا يعتبر الخطاط خطاطا إلا إذا أتقنه وهو أصعب الخطوط ، وأول من وضع قواعده الوزير

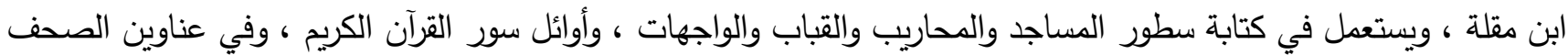

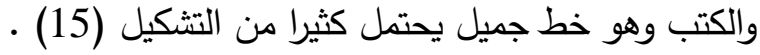

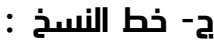

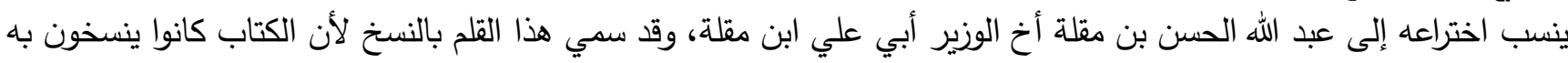

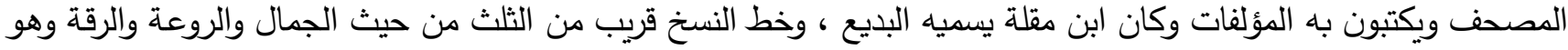

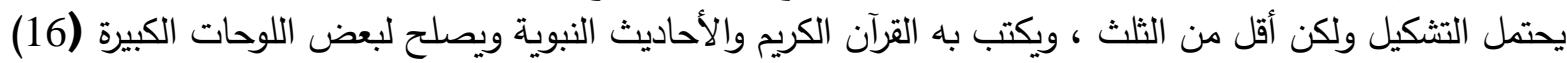

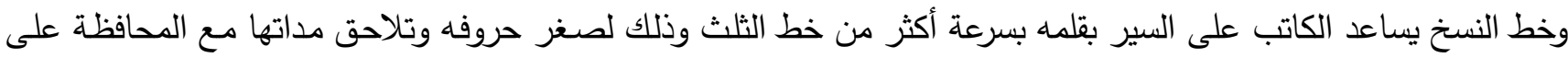

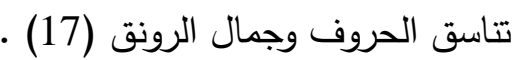

د- الخط الإجازة : الإق

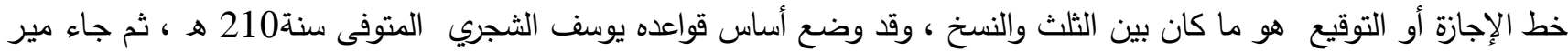

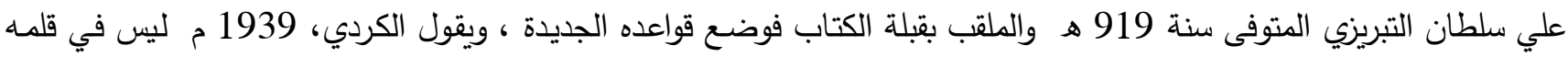

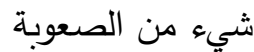

ولايحتاج الكاتب لكثرة التمرين فيه ليرسخ في الذهن كيفية المزج والخلط بين الثلث والنسخ (18).

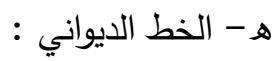

سمي هذا الخط بالّيواني لاستعماله في الديوان العثماني الهمايوني السلطاني وأول من وضع قواعده إبراهيم منيف ، بعد فتح السلطان

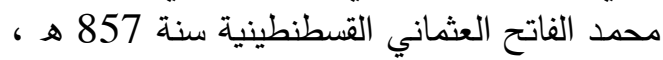

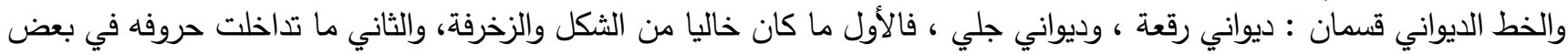

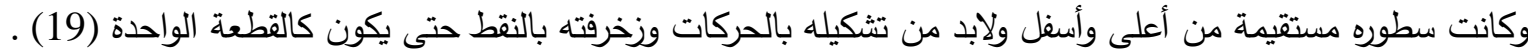

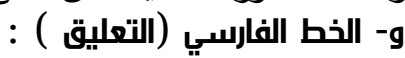
ورد أن حسن فارسي كاتب عضدا الفد الدولة استتبط قواعد خط التعليق الأول من أقلام النسخ والرقاع والثلث ، ويسمى الخط الفارسي

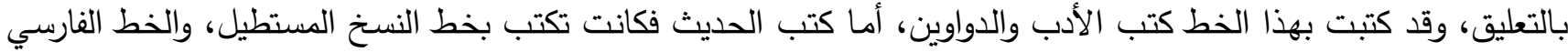

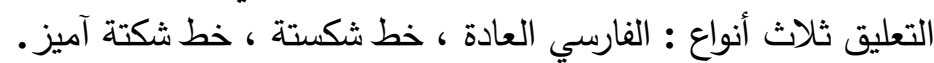

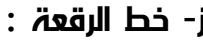
هو من الخطوط المتأخرة المستحدثة قيل اخترعه و وضع قواعده ممتاز بك مصطفى أفندي المستثار حوالي سنة 1280 هـ (20)

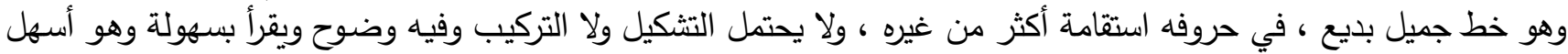

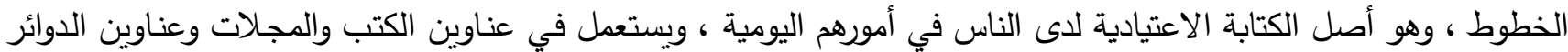

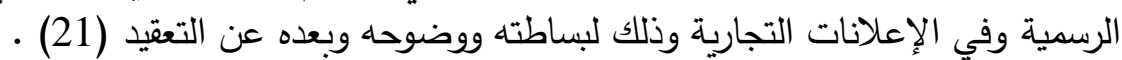

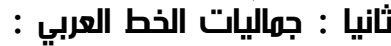

إن فن الخط العربي فن مستقل له منطقا جماليا، تحكمه خصائصه وأساليبه ومساراته، فجماليات اللوحة الخطية ليست في جمالية

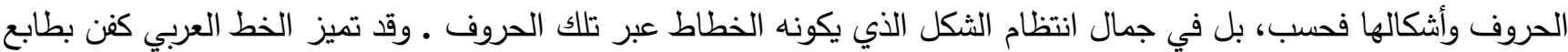

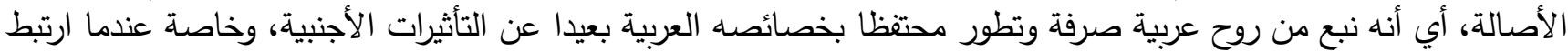

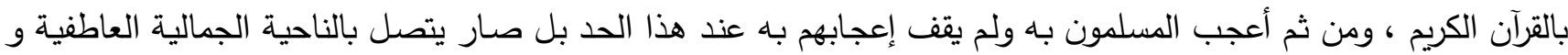

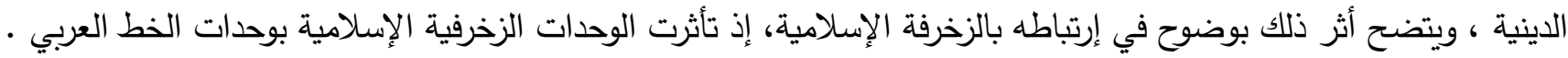

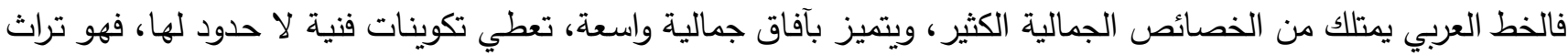

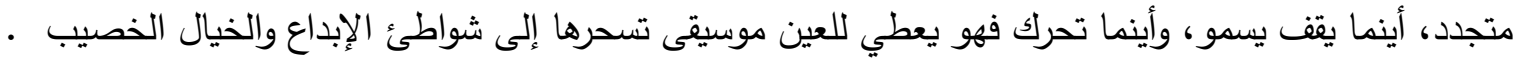

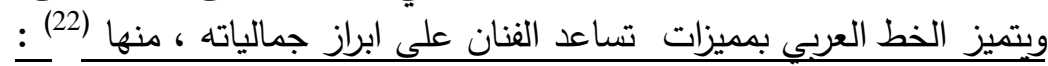

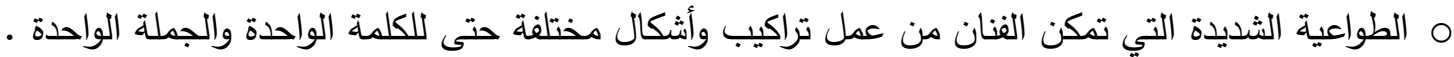

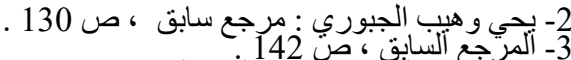

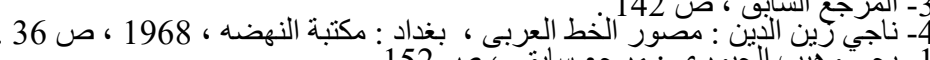

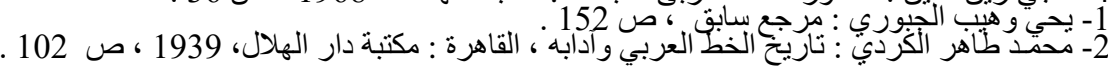

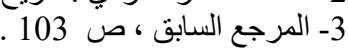

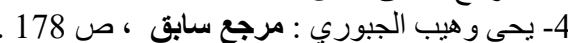
22 ـ صبحي الثاروني : الحرف العربي في التصوير الحديث وأصوله في التراث ، مجلة فكر وفن ، سويسر ا : دار ألبرت تايلا للنشر ، العدد (33) ، 1979 ، 
م الارتفاعات والاستدارات وقدرتها على الاطالة والتمطيط .

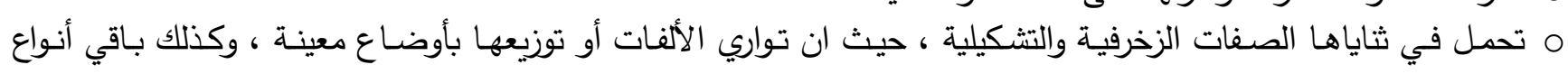

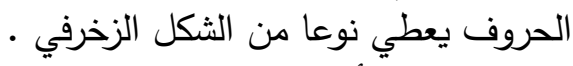

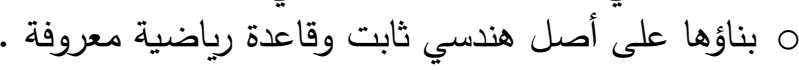

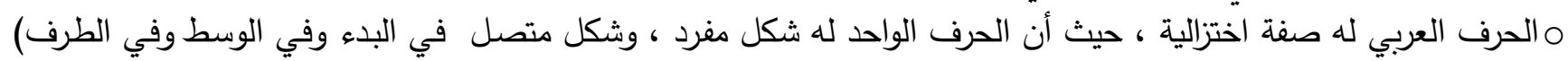

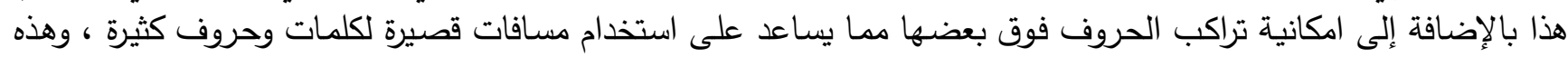

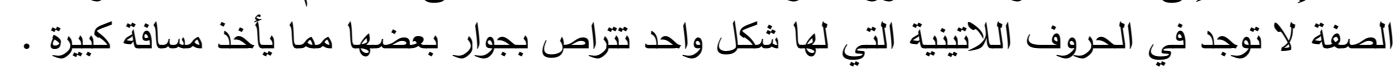

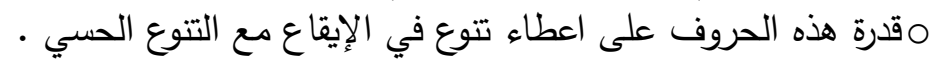

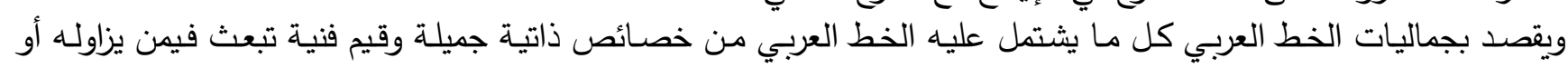

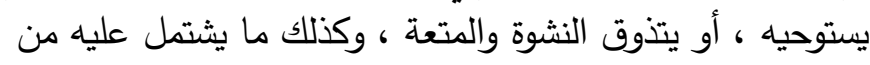

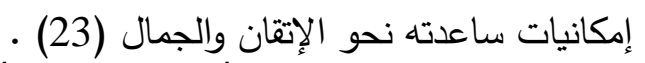

وترجع جماليات الخط العربيات إلى أمور عديدة كان الجان أهمها أمران أساسيان:

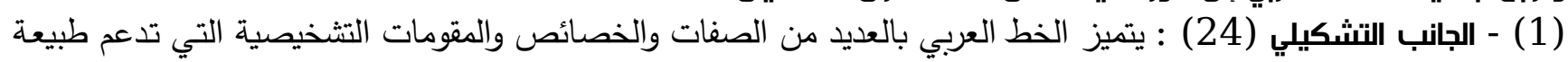

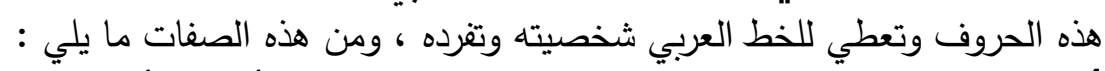

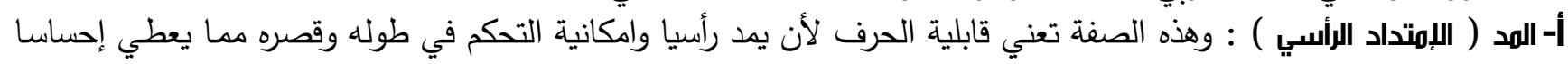

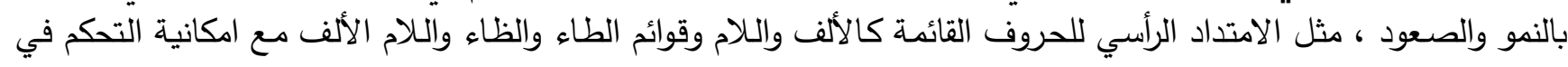
بالب- البسط ( الاهتداد الأفقي ) : ويعني مد أجزاء الحروف الأفقية ، كبسط الياء والسين والصاد والكاف مما يعطي احساسا بالاستقرار والاتزان في شكل الحروف

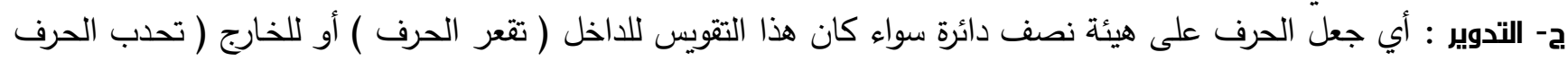

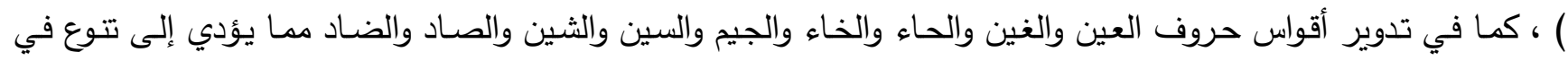

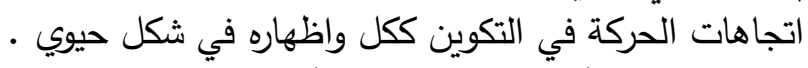

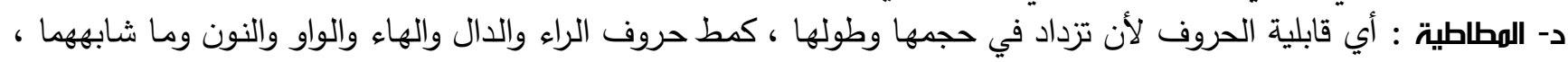

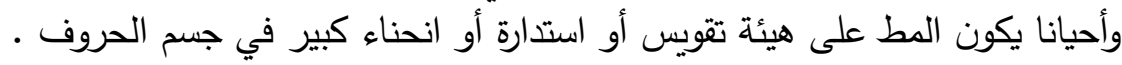

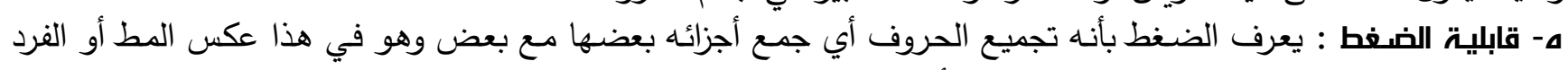

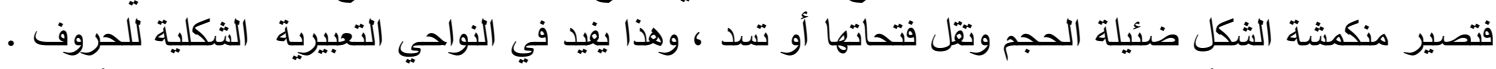

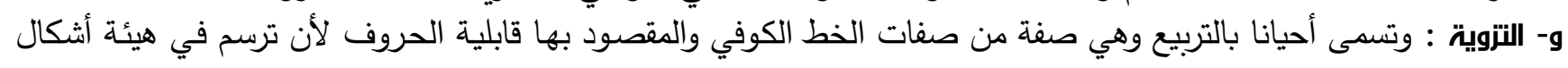

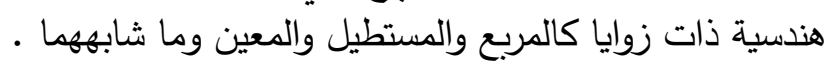

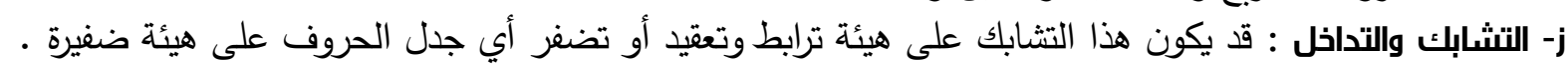

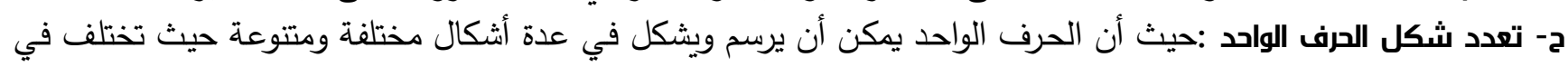

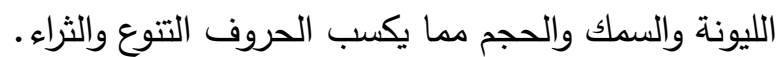

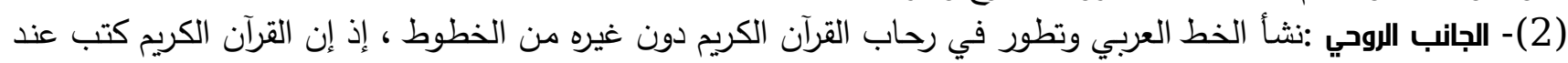

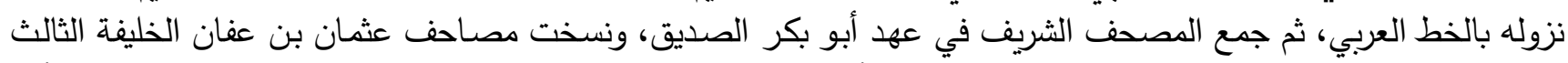

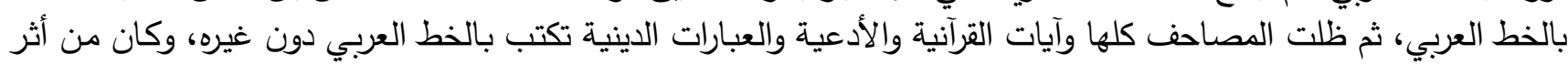

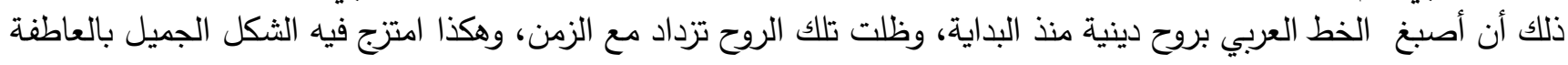

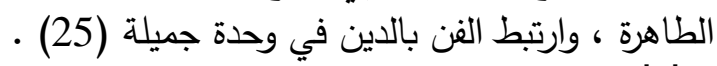
ثالثا : الإستخدامات النفعية النفاين في اللخط العربي :

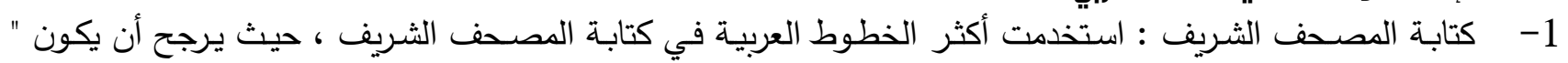

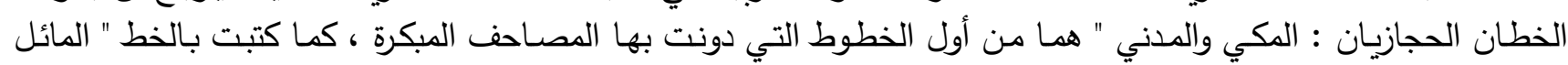

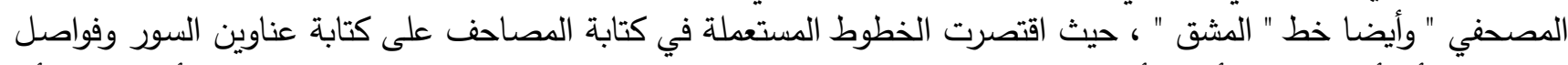

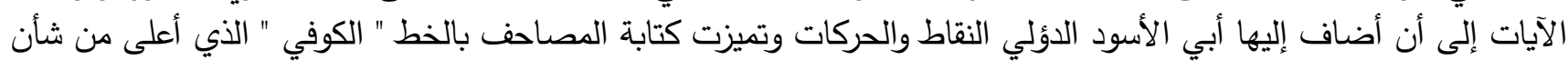
الخط واجلاله . 


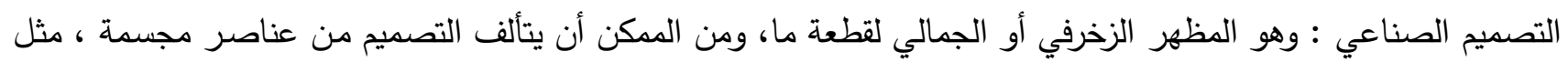

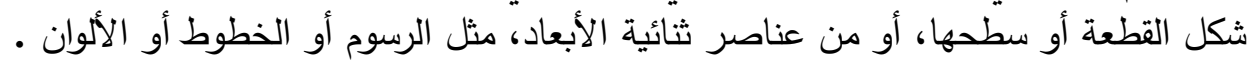

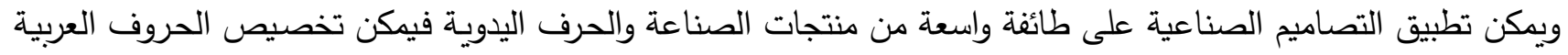

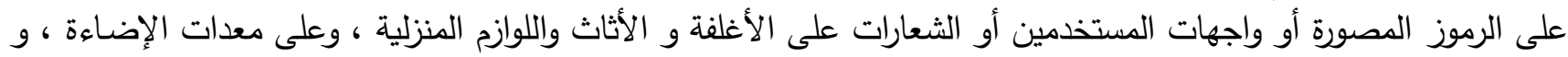

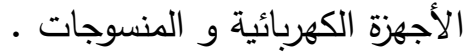

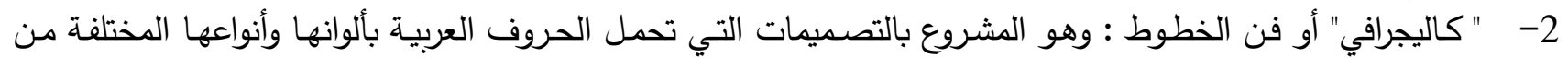
الخط الكوفي أو خط الرقعة وبعض الجمل فالت فاللغة الفارسية ، وغيرها من اللغات التي تستخدم الحروف العربية ، وهو شكل جديد 0 من التصميم يجسد الطابع العربي التوفي 3- زخارف المنسوجات : بإدخال تعديلات وتحسينات مختلفة على ملابس متنوعة وخاصـة باختلاف المواقف والمناسبات،

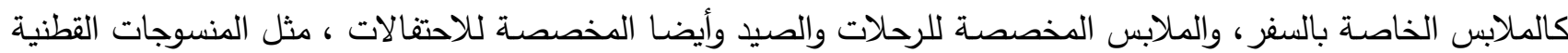
المطبوعة، المنسوجات الكتانية المطبوعة ، المنسوجات الحريرية التي تستخدام خيوط الحرير حول الرئل الرقبة وفى نهاية الأكمام وذيل

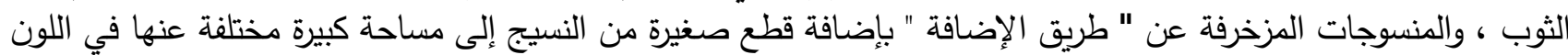

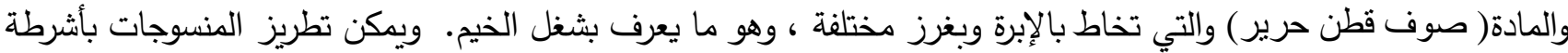

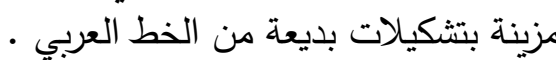

4- في العمارة والفراغات الداخلية الإسلامية : استخدم الخط العربي في العمارة والفراغات الداخلية التقليدية استخدامات عديدة

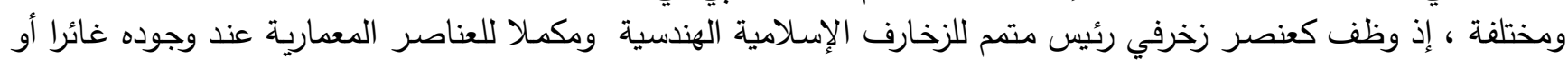
نافرا من خلال الظلال الناتجة ليوظف كعنصر تذكيري ، وكذلك ليصبح عنصرا معبرا عن الحضارة العربية الإسـلامية ببعدها

ويتجلى أوضح استخدام للخط العربي في العمارة الدينية ( المساجد والمدارس وغيرها ) ، ويظهر بشكل خاص في واجهاتها

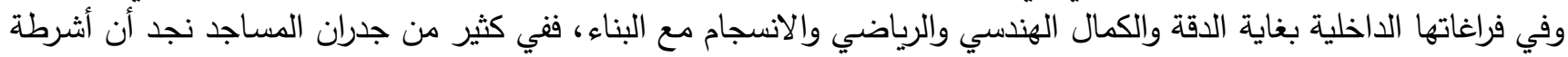

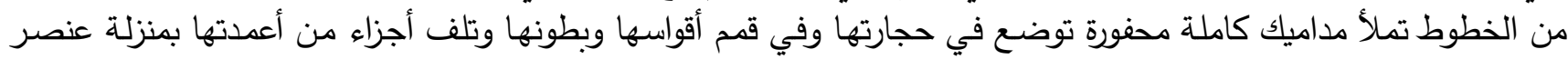

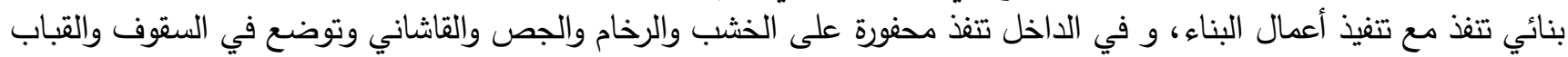

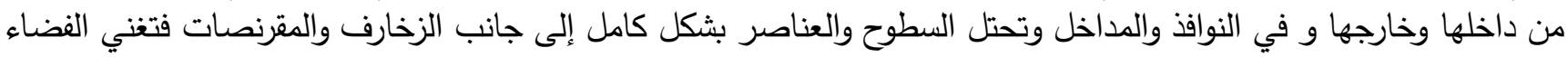

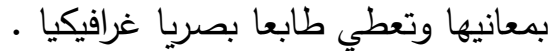
5- الزخارف والكتابات الخزفية : تأتي مرحلة تنفيذ الزخارف الإسـلامية والحروف العربية على الخزف عند إجراء الجاء البطانات

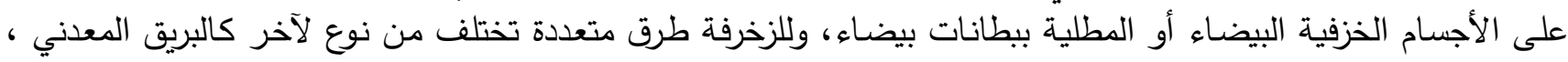

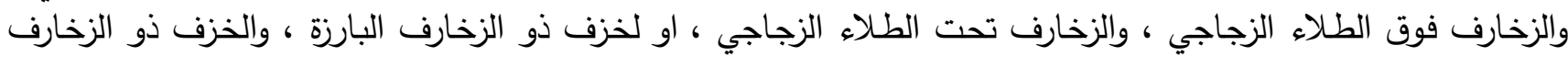

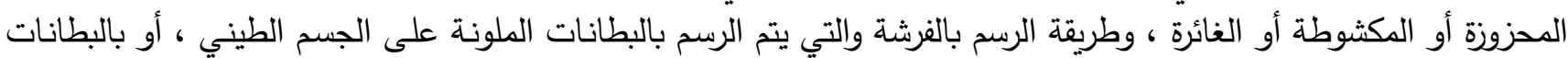

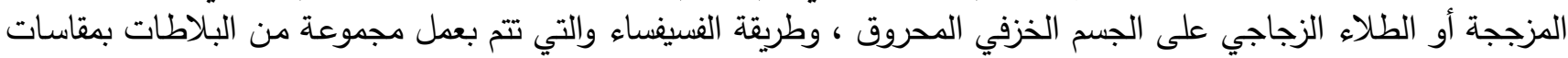

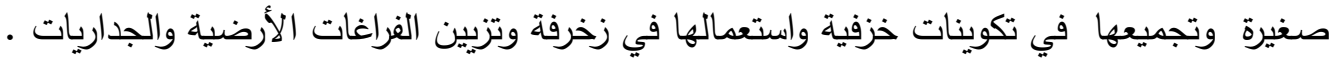

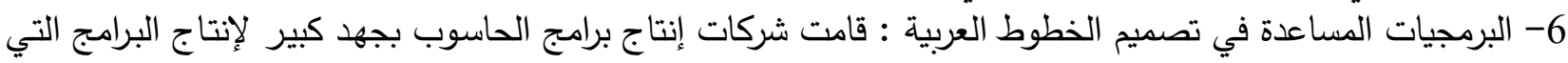

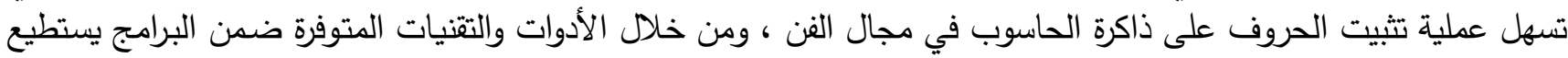

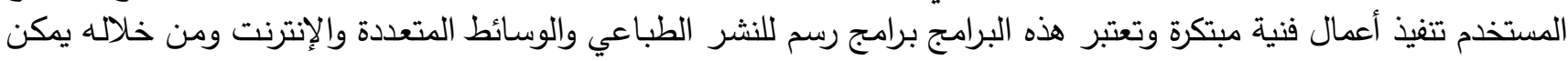

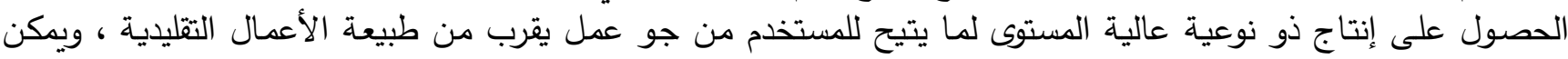

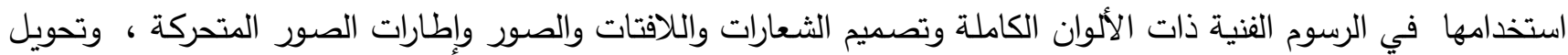

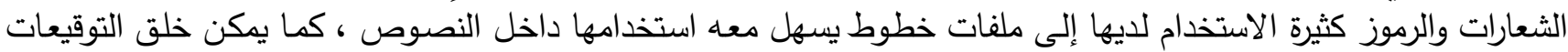

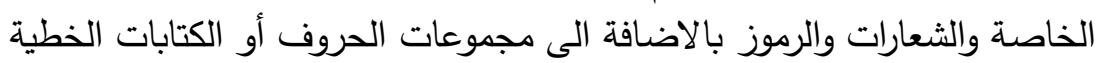

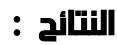

في ضوء ما تم عرضه من إطار نظري في الدراسة ، وملاحظة النماذج التي مارسها طلاب مقرر ( فنون ) بقسم التربية الفنية

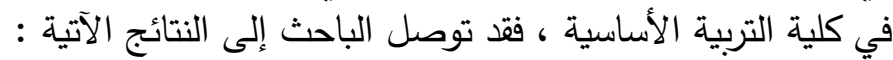
1- يمكن توظيف الخط بأنواعه سواء كوفي أو نسخ أو ثلث أو ديواني أو فارسي لزيادة جماليات الشكل وتحقيق قيمة جمالية مضافة .

2- تضيف الكتابات العربية خاصة تلك المقتبسة من القرآن الكريم أو الحديث النبوي الثريف قيما معنوية وتعبيرية .

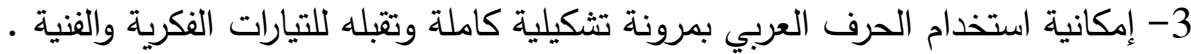


4- تتميز الخطوط العربية بالجمال والروعة ومعظمها يحتمل التشكيل الذي يزيدها روعة وجمال.

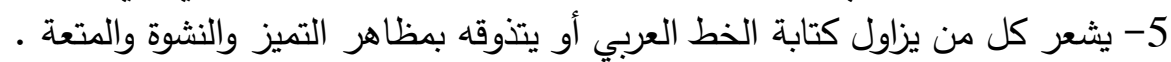

التوميات :

1- يوصسى الباحث بضئ بـرورة التوجه للمزيد من الاهتمام بالخطوط العربية باعتبارها مجالا واسع الإبداع وما تحمله من قيم

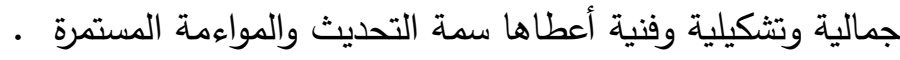

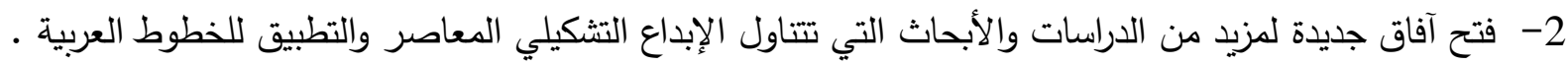

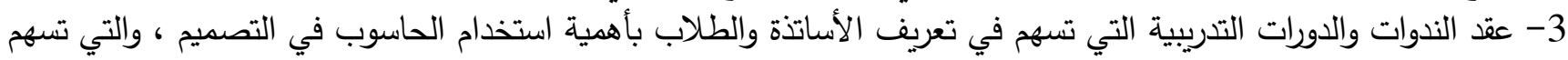

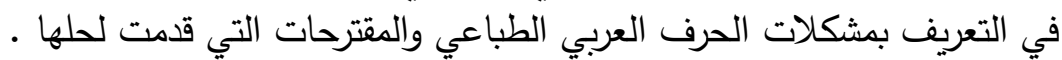

المراجع

1 - ابراهيم جمعه : دراسة في تطور الكتابات الكوفية على الأحجار في مصر في القرون الخمسة الأولى للهجرة ، القاهرة :

المطبعة العالمية ، 1969 .

2-- حسن الباشا: موسوعة العمارة والآثار والفنون الإسلامية ، الجزء الثالث ، بيروت : أوراق شرقية للطباعة والنشر، 1999 ، 1969 ـ

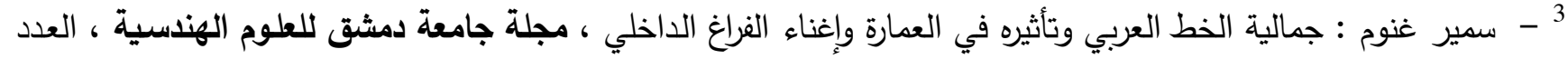
الثاني ، 2014 ( 2014

4- صبحي الثاروني : الحرف العربي في التصوير الحديث وأصوله في التراث ، مجلة فكر وفن ، سويسرا : دار ألبرت تايلا

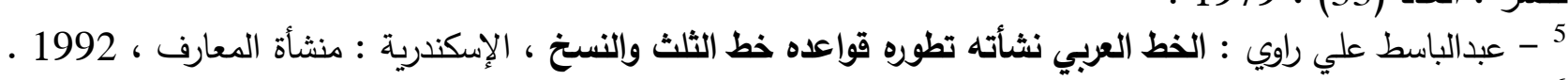

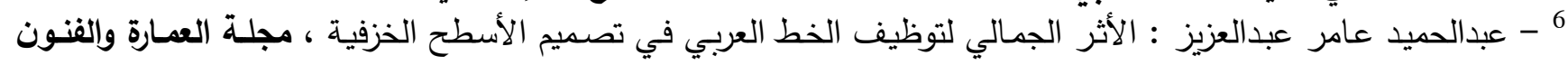
والعطوم الإنسانية ، ، العدد (3) ، 2016 ، 2016 . 7 - عبد المحسن حسين عبد الرضانسا شيشتر : الوظيفة الزخرفية للحرف العببي كمدخل تجريبي لتدريس التصميم في التربية

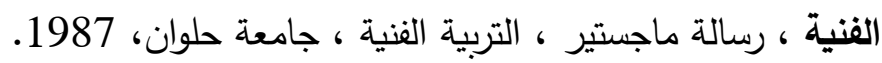
8- عبد المنعم خيري العاني : تصميم برنامج للإبداع في الخط العربي الكوفي، أطروحة دكتوراه ، كلية الفنون الجميلة ، جامعة بغداد ، 1995 . 199

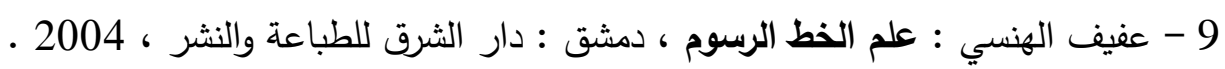

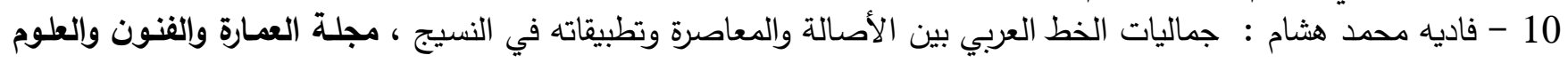

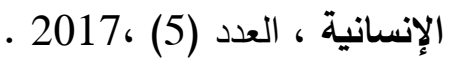
11- فوزي سالم عفيفي : نشأة وتطور الكتابة الخطية العربية ودورها الثقافي والاجتماعي ، الكويت : وكالة المطبوعات ، . 1980

12- محمد طاهر الكردي : تاريخ الخط العربي وآدابه ، القاهرة : مكتبة دار الهلال، 1930 ـ 1939 .

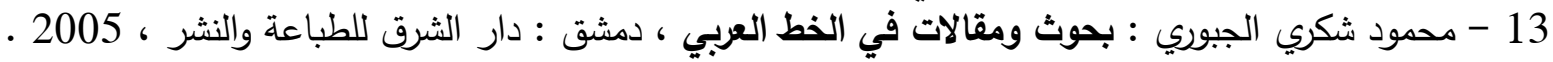

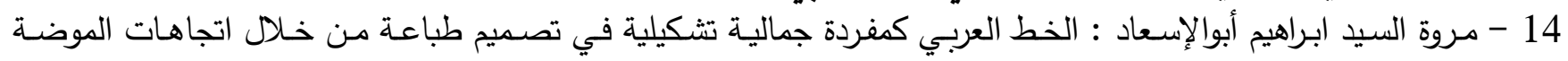

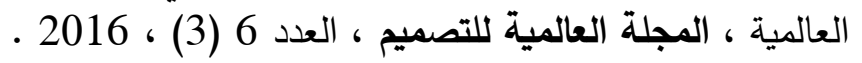

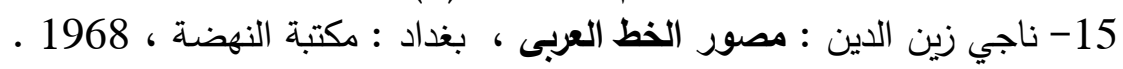

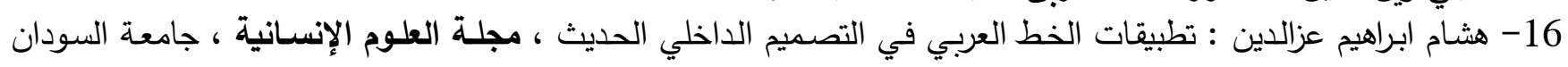

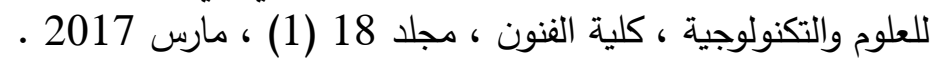
17- يحي وهيب الجبوري : الخط والكتابة في الحضارة العربية ، بيروت : دار الكية الغرب الاسلامي ، 1994 ، 